\title{
COMMUNICATION
}

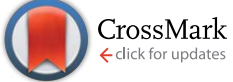

Cite this: RSC Adv., 2016, 6, 56134

Received 26th April 2016 Accepted 6th June 2016

DOI: $10.1039 / c 6 r a 10759 a$

www.rsc.org/advances

\section{Primaquine-based ionic liquids as a novel class of antimalarial hits $\uparrow$}

\author{
Ricardo Ferraz, ${ }^{\star a b}$ Joana Noronha, ${ }^{a}$ Fernanda Murtinheira, ${ }^{c}$ Fátima Nogueira, ${ }^{c}$ \\ Marta Machado, ${ }^{d}$ Miguel Prudêncio, ${ }^{d}$ Silvia Parapini, ${ }^{e}$ Sarah D'Alessandro, ${ }^{e}$ \\ Cátia Teixeira, ${ }^{a}$ Ana Gomes, ${ }^{a}$ Cristina Prudêncio ${ }^{\text {bf }}$ and Paula Gomes ${ }^{\star a}$
}

Ionic liquids derived from active pharmaceutical ingredients may open new perspectives towards low-cost rescuing of classical drugs. Thus, we have synthesized novel ionic liquids derived from the antimalarial drug primaquine, and evaluated them in vitro against three stages of malaria parasites. Results from this unprecedented approach open a new chapter in the history of antimalarial drugs.

ILs are organic salts (with at least one organic cation), generally with melting points below $100{ }^{\circ} \mathrm{C}$ (some of them are liquid at room temperature) ${ }^{1,2}$ Recently, the most promising applications for ILs seem to be their use as active pharmaceutical ingredients (APIs), as the pharmaceutical industry is starting to pay attention to the potential benefits of their non-crystalline forms, among other potentially beneficial properties. ${ }^{2,3}$ In fact, ILs could avoid several problems associated with solid forms of drugs, such as polymorphic conversion, low solubility, low bioavailability for crystalline solids, and the tendency of amorphous forms to spontaneously crystallize. ${ }^{2}$ Thus, ILs might contribute to rescuing "old" drugs, whose clinical application has been, or is being, gradually abandoned, due to unfavorable

${ }^{a}$ UCIBIO-REQUIMTE, Departamento de Quimica e Bioquímica, Faculdade de Ciências da Universidade do Porto, Rua do Campo Alegre 687, 4169-007 Porto, Portugal. E-mail:pgomes@fc.up.pt

${ }^{b}$ Ciências Quimicas e das Biomoléculas, CISA, Escola Superior de Tecnologia da Saúde do Porto - Instituto Politécnico do Porto, Rua Valente Perfeito 322, 4400-330 Vila Nova de Gaia, Portugal. E-mail: ricardoferraz@eu.ipp.pt

${ }^{c}$ Global Health and Tropical Medicine, GHTM, Instituto de Higiene e Medicina Tropical, IHMT, Universidade Nova de Lisboa, UNL, Rua da Junqueira 100, 1349oos Lisboa, Portugal

${ }^{d}$ Instituto de Medicina Molecular, Faculdade de Medicina Universidade de Lisboa, Av.Prof. Egas Moniz, 1649-028 Lisboa, Portugal

${ }^{e}$ Dipartimento di Scienze Farmacologiche e Biomolecolari, Università degli Studi di Milano, Via Pascal, 3620133 Milano, Italy

${ }^{f} I 3 S$ - Instituto de Investigação e Inovação em Saúde, Universidade do Porto, Rua Alfredo Allen, 208, 4200-135 Porto, Portugal

$\dagger$ Electronic supplementary information (ESI) available. See DOI: $10.1039 /$ c6ra10759a absorption-distribution-metabolism-excretion-toxicity (ADMET) profiles.

Since most available APIs are found in the cationic or anionic form, they can be combined with either an inert counterion or a counterion displaying additional biological properties of interest, ${ }^{2-7}$ to create novel ILs-APIs. In fact, most ionic antimalarial APIs are administered as salts (e.g., primaquine bisphosphate, proguanil hydrochloride, sodium artesunate), and the formulation and formation of these salts are considered essential steps in the preclinical phase of modern drug development. ${ }^{3}$

The synthesis of ILs-APIs has been proven possible by different methods, such as metathesis and acid-base neutralization, each of which with its advantages and disadvantages. ${ }^{3,8}$ Recently, we have developed a method termed neutralization with buffer, ${ }^{1}$ which avoids contamination problems associated to the metathesis approach, ${ }^{\mathbf{1}}$ where anionic ampicillin was combined with six different organic cations., ${ }^{1,7}$ This type of combination showed that ILs-APIs physicochemical descriptors like solubility and lipophilicity could be tuned by choice of the adequate cation. ${ }^{7}$ Furthermore, the ampicillin-based ILs developed showed activity against antibiotic-resistant Gram-negative bacteria. ${ }^{6}$

Despite massive efforts to fight malaria, according to the World Health Organization, in 2015 half a million people were killed, roughly a quarter billion got infected and nearly half of the population was at risk of malaria infection, in most cases by the Plasmodium falciparum (P. falciparum) species., ${ }^{\mathbf{9 1 0}}$ Research in the field of antimalarial chemotherapy has been a neverending quest for the next miracle antiplasmodial drug, due to fast emergence of resistant parasite strains. Therefore, ILs-APIs could be regarded as a potential approach to bypass the resistance issues that rapidly emerge even for novel highly potent APIs. ${ }^{11-14}$ Moreover, new antimalarial approaches should be made available at low-cost, or else they will barely be of real use in malaria-endemic regions. Thus, based on recent findings regarding low-cost production of ILs-APIs by combining classical drugs with opposed polarities, ${ }^{3,6,7,15}$ it is reasonable to assume that development of ILs derived from classical 


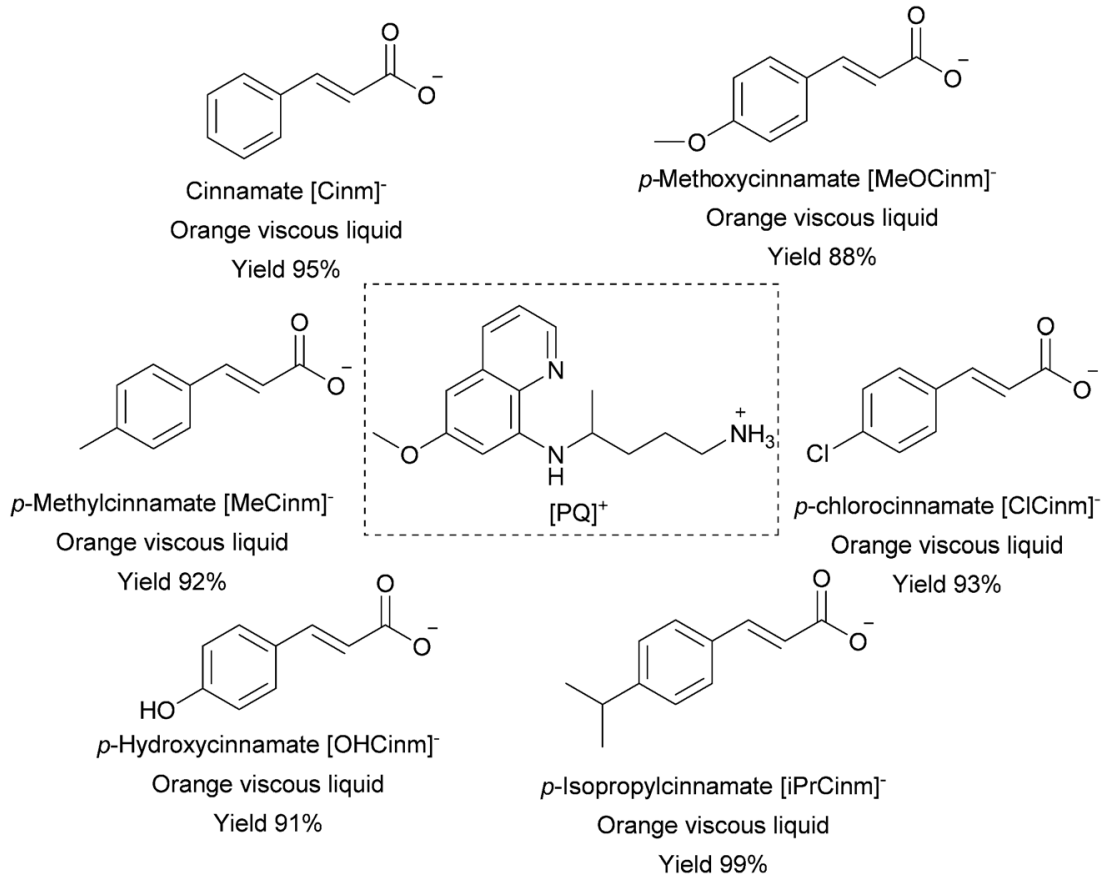

Fig. 1 Representation of the prepared API-ILs, where cationic primaquine [PQ] ${ }^{+}$(inside dashed rectangle at the center) was combined with different cinnamates, as counterions, to form novel primaquine-derived ILS. Synthesis (neutralization method, see text and Fig. 2) yields and physical features of the produced ILs are also provided.

antimalarial drugs emerges as an innovative and cost-effective strategy to overcome the problems associated with emblematic antimalarials. ${ }^{\mathbf{1 0 , 1 6}}$ Hence, the present work addresses the chemical synthesis and in vitro evaluation of novel ILs based on primaquine, an approved antimalarial aminoquinoline whose basic character allows it to be used as the organic cation in new ILsbased formulations (Fig. 1). It was envisaged that this cationic antimalarial could be combined with different carboxylates (Fig. 1), whose choice was based on their interest regarding antimalarial properties..$^{\mathbf{1 0 , 1 3 , 1 6 - 1 8}}$ We were particularly interested in exploring the behavior of cinnamate-derived ILs, as cinnamic acids were previously used by us to prepare $N$-cinnamoyl derivatives of the same drug, inspired by the Meunier's covalent bitherapy concept, ${ }^{19}$ producing covalent conjugates with improved in vitro activities against liver-stage malaria parasites. ${ }^{\mathbf{1 6}}$ Interestingly, their new ionic counterparts reported herein were active in vitro against liver-stage forms, asexual blood-stage forms, and gametocytes of Plasmodium parasites, confirming the novel ILs as triple-stage antimalarial hits.

\section{Results and discussion}

\section{Synthesis and characterization}

The traditional production of ILs involves a metathesis reaction of ion halide with an adequate anion..$^{20,21}$ Problems associated to this method include use of halo-organic solvents like dichloromethane, and difficulties in obtaining pure ILs. In view of this, we decided to use instead the neutralization method, by simply reacting the basic antimalarial drug with equimolar amounts of the carboxylic acid of interest (Fig. 2). This allowed the production of the target ILs free of impurities, as confirmed by structural analysis (see below).

All products were obtained in high yields as viscous liquids (Fig. 1), and were fully characterized by high resolution mass spectrometry (HRMS) and by ${ }^{1} \mathrm{H}$ and ${ }^{13} \mathrm{C}$ nuclear magnetic resonance (NMR). Experimental details and spectroscopic data on ILs-APIs prepared are given as ESI. $\dagger$

${ }^{1} \mathrm{H}$ NMR analysis not only allowed to check for the high purity of the isolated antimalarial-based ILs, which was

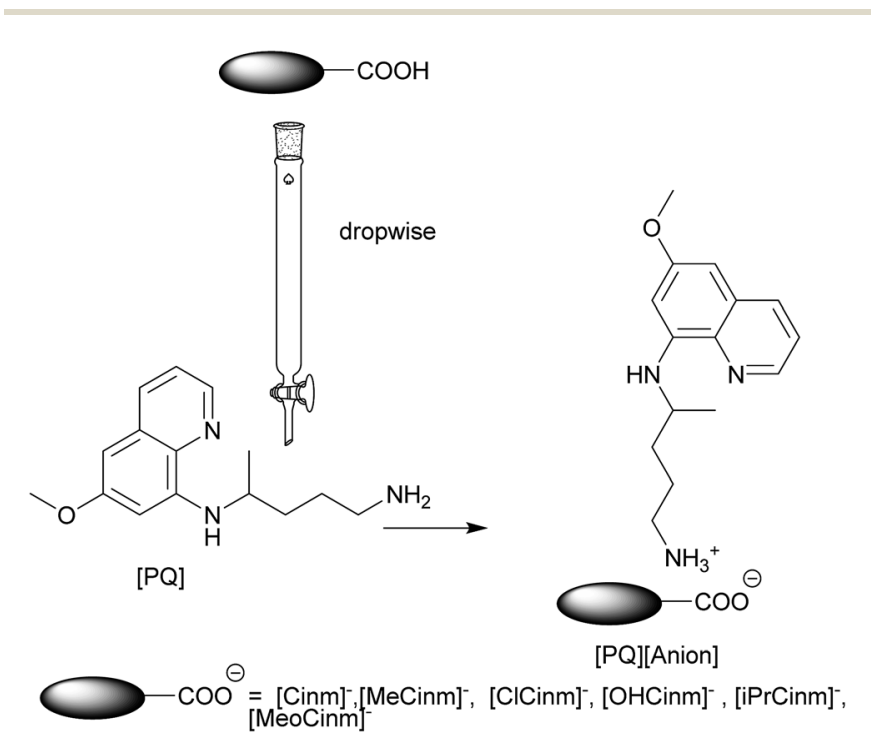

Fig. 2 Schematic synthetic procedure for the preparation of primaquine-based ILs. 
confirmed by HRMS, but also to confirm the (i) proton transfer from the carboxylic acid group to the aliphatic amine, and (ii) expected cation/anion 1:1 ratio through quantitative integration of characteristic ${ }^{1} \mathrm{H}$ resonance peaks. NMR studies also provided evidence that all ILs produced were stable over time (data not shown).

\section{Antiplasmodial activity - blood stage}

Seven decades after its discovery, primaquine remains the only antimalarial drug of worldwide clinical use that (i) eliminates dormant liver forms of Plasmodia (hypnozoites), responsible for recurrent disease relapses, especially in vivax malaria-endemic regions, (ii) blocks disease transmission due to its gametocytocidal action, and (iii) has not raised any clinically relevant resistance issues thus far. ${ }^{10,22}$ Still, the therapeutic value of primaquine is overshadowed by toxicity issues, especially to persons with glucose-6-phosphate dehydrogenase (6GPD) deficiency, and by the drug's poor activity against blood-stage parasites. ${ }^{10}$ Hence, primaquine-based structures with improved blood-stage activity should be continuously pursued. Bearing this in mind, we started by evaluating the new ILs in vitro against erythrocytic forms of chloroquine-sensitive (3D7) and chloroquine-resistant (Dd2) P. falciparum strains (Table 1). Interestingly, all ILs assessed displayed higher activity than primaquine against the parasites' blood stages. Our results show that all ILs tested have a lower $\mathrm{IC}_{50}(70 \%$ lower, on average) than the reference value reported for primaquine by Sykes et al. ${ }^{23}$ for the erythrocytic stages of P. falciparum strain 3D7 $(6.09 \mu \mathrm{M})$. A similar trend was observed for the chloroquine-resistant Dd2 strain, where $\mathrm{IC}_{50}$ values were reduced by as much as $75 \%$ (0.69 $\mu \mathrm{M}$, for $[\mathrm{PQ}][\mathrm{ClCinm}])$, as compared to the value reported for primaquine against this strain $(4.70 \mu \mathrm{M}){ }^{24}$

Notably, previous work from our group, where primaquine was reacted with cinnamic acids to produce the corresponding covalent (amide) conjugates (structures 1 on Fig. 3), showed that these conjugates were poorly active against blood-stage $P$. falciparum parasites, as only one (1) in Fig. 3) of the eleven conjugates then assayed displayed an $\mathrm{IC}_{50}$ value below $10 \mu \mathrm{M}$ $(4.84 \mu \mathrm{M}){ }^{16}$ Hence, we now demonstrate that the simple

Table 1 Blood-stage antiplasmodial activity against chloroquinesensitive strain 3D7 and chloroquine-resistant strains Dd2 of P. falciparum

\begin{tabular}{lll}
\hline & \multicolumn{2}{l}{ Strain } \\
\cline { 2 - 3 } & \multicolumn{3}{l}{$3 \mathrm{D} 7$} & $\mathrm{Dd} 2$ \\
\cline { 2 - 2 } Compound & $\mathrm{IC}_{50} / \mu \mathrm{M}$ & $\mathrm{IC}_{50} / \mu \mathrm{M}$ \\
\hline $\mathrm{PQ}$ & $6.09 \pm 0.38^{a}$ & $4.70 \pm 0.06^{b}$ \\
{$[\mathrm{PQ}][$ Cinm $]$} & $1.13 \pm 0.04$ & $1.38 \pm 0.04$ \\
{$[\mathrm{PQ}][$ MeCinm $]$} & $0.94 \pm 0.28$ & $1.40 \pm 0.12$ \\
{$[\mathrm{PQ}][$ OHCinm $]$} & $1.68 \pm 0.88$ & $1.18 \pm 0.13$ \\
{$[\mathrm{PQ}][$ MeOCinm $]$} & $1.26 \pm 0.03$ & $1.16 \pm 0.24$ \\
{$[\mathrm{PQ}][$ iPropCinm $]$} & $3.40 \pm 0.30$ & $1.08 \pm 0.03$ \\
{$[\mathrm{PQ}][$ ClCinm $]$} & $1.56 \pm 0.41$ & $0.69 \pm 0.04$
\end{tabular}

${ }^{a}$ Values taken from Sykes et al. ${ }^{23 b}$ Values taken from Gorka et al. ${ }^{24}$

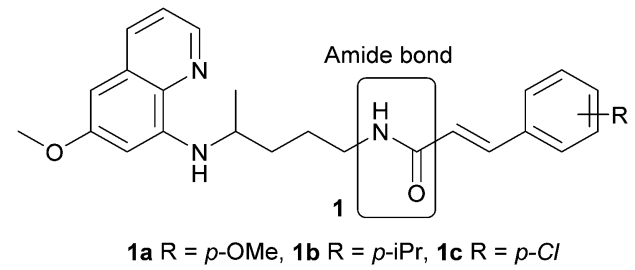

Fig. $3 \mathrm{~N}$-Cinnamoyl-primaquine conjugates previously reported by us. ${ }^{16}$

conversion of the covalent amide into the ionic ammonium carboxylate bond between the primaquine and the cinnamic acid moieties has a clear positive impact on blood stage activity. In view of this, primaquine-based ILs may constitute a remarkable first step towards low-cost rescuing and improvement of this classical antimalarial, and related antimalarial 8-aminoquinolines.

\section{In vitro liver stage activity}

The positive effect of producing primaquine-based ILs on blood-stage activity, described above, might be hampered if any of the other antimalarial attributes of primaquine were dramatically affected. Hence, to check whether the reported ILs preserved the liver-stage activity of the parent drug, we selected three primaquine-based ILs, [PQ][MeOCinm], [PQ][iPropCinm] and $[\mathrm{PQ}][\mathrm{ClCinm}]$, and their covalent counterparts 1a-c, respectively (Fig. 3), for additional in vitro screening against liver-forms of $P$. berghei parasites, as well as toxicity to Huh7 human hepatoma cells ${ }^{25}$ (Fig. 4). Results were compared to those previously reported for covalent analogues 1a-c, obtained under identical conditions. ${ }^{10}$

Cell confluency measurements (circles) depicted on Fig. 4 show that all tested ILs are non-toxic to human hepatocytes. Regarding activity against liver forms of $P$. berghei (Fig. 4, bars), the ILs displayed dose-dependent responses that were better than, for $[\mathrm{PQ}][\mathrm{MeOCinm}]$ and $[\mathrm{PQ}][\mathrm{iPropCinm}]$, or equivalent to, for $[\mathrm{PQ}][\mathrm{ClCinm}]$, that of the reference drug primaquine. Though being slightly less active than their respective covalent conjugates against liver-stage parasites ${ }^{10}$ the new antimalarial ILs were confirmed to display improved blood-stage antimalarial activity without any loss of liver-stage activity as compared to parent primaquine.

\section{Antiplasmodial activity - gametocyte stage}

The two most active primaquine-derived ILs against liver-stage parasites, $[\mathrm{PQ}][$ MeOCinm] and $[\mathrm{PQ}][$ iPropCinm $]$, were (along with their covalent counterparts) further evaluated against 3D7 transgenic gametocytes ${ }^{27}$ using primaquine as reference drug (Table 2).

Results displayed on Table 2 show that the primaquinederived ILs tested have gametocytocidal activities equivalent or superior to those of their covalent $\mathrm{N}$-cinnamoyl-primaquine counterparts, 1a,b, and to that of the parent drug, primaquine. The most significant effect is observed when comparing both compounds derived from $p$-isopropylcinnamic acid, i.e., 


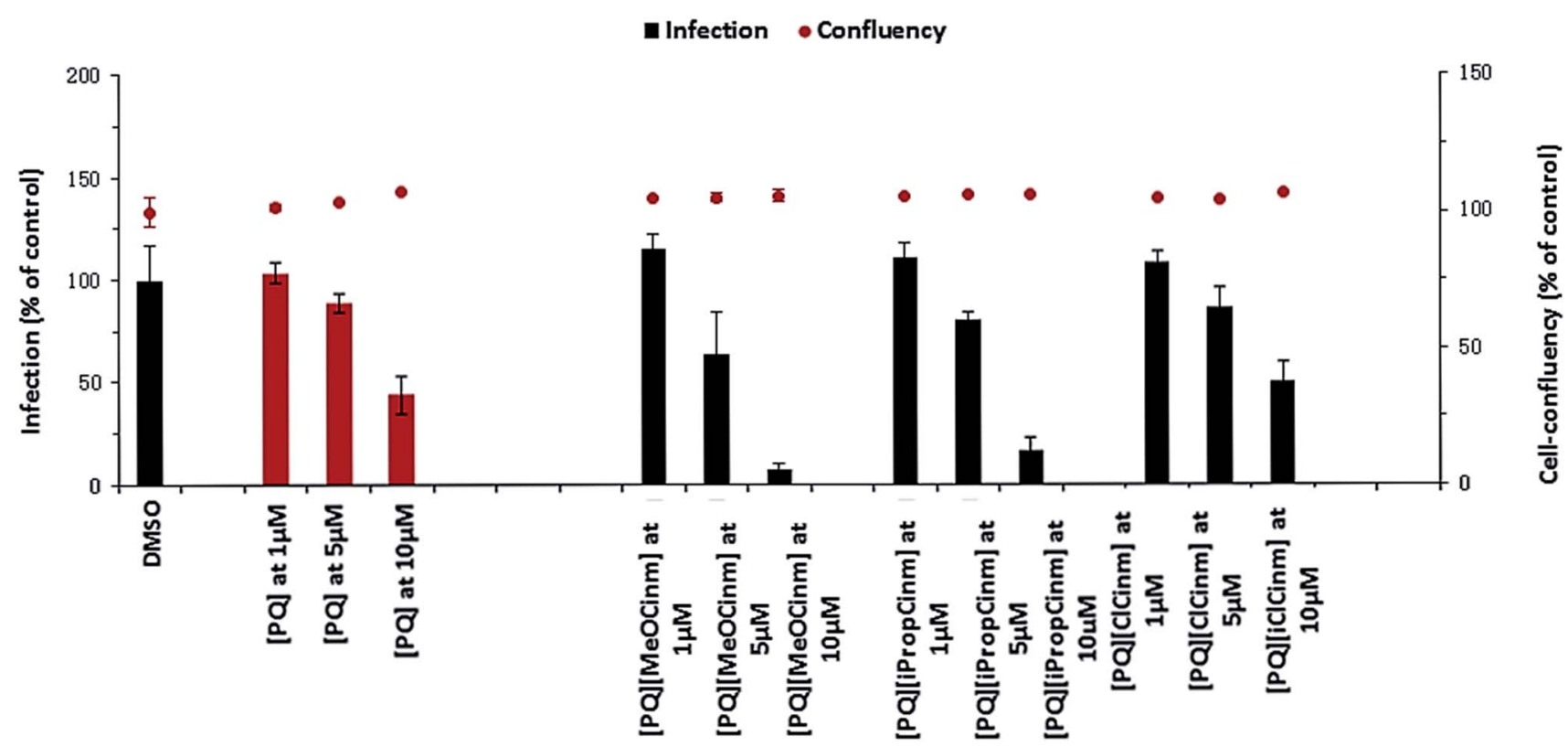

Fig. 4 Activity of [PQ][MeOCinm], [PQ] [iPropCinm] and [PQ][ClCinm] against $P$. berghei liver stages. Anti-infective activity (infection scale, bars) and toxicity to hepatoma cells (cell confluency scale, circles) are shown. Primaquine was included for comparison. Infection loads of Huh7 cells, a human hepatoma cell line, were determined by bioluminescence measurements of cell lysates $48 \mathrm{~h}$ after infection with luciferase-expressing P. berghei parasites. ${ }^{26}$

Table 2 In vitro antimalarial activity against stage $\mathrm{V}$ Plasmodium falciparum gametocytes from a 3D7 transgenic line

\begin{tabular}{llll}
\hline Compound & $\mathrm{IC}_{50} / \mu \mathrm{M}$ & $\operatorname{clog} P^{a}$ & $\operatorname{clog} S^{a}$ \\
\hline$[\mathrm{PQ}][$ MeOCinm $]$ & $18.4 \pm 2.9$ & 2.52 & -4.65 \\
{$[\mathrm{PQ}][$ iPropCinm $]$} & $10.1 \pm 2.5$ & 3.64 & -6.10 \\
$\mathbf{1 a}$ & $16.5 \pm 4.4$ & 4.66 & -5.05 \\
$\mathbf{1 b}$ & $>20.0$ & 5.78 & -6.50 \\
Primaquine & $19.7 \pm 4.8$ & 2.78 & -2.24
\end{tabular}

${ }^{a}$ Descriptors values estimated with molecular operating environment (MOE) software. ${ }^{28}$

[PQ][iPropCinm] and $\mathbf{1 b}$, as the former displayed an in vitro gametocytocidal activity over 2-fold higher than the latter. Eventually, observed differences can be ascribed to lipophilicity/water-solubility issues; as expected, the ionic primaquine-derived ILs are associated to $\operatorname{lower} \operatorname{clog} P$ and higher $\operatorname{cog} S$ values than their respective covalent counterparts 1a,b (Table 2), whereas differences between both ILs can only be attributed to their anionic portion.

All in all, irrespective of structure-activity relationships underlying experimental observations, it is obvious that the replacement of the amide bond, as in covalent conjugates $\mathbf{1 a}, \mathbf{b}$, by its ionic ammonium carboxylate counterpart, as in the ILs tested, not only conserves but may even slightly improve antimalarial performance at the gametocytocidal activity level.

\section{Concluding remarks}

In conclusion, the present work reports a new low-cost and efficient method for the synthesis of ILs based on the classical antimalarial drug primaquine. Overall, a total of six novel ILs were synthesized and screened against blood-stage chloroquine-sensitive and chloroquine-resistant $P$. falciparum parasites, and found to exhibit improved in vitro performances as compared to their parent drug. A subset of such ILs was further evaluated regarding cytotoxicity and activity against both liver-stage $P$. berghei parasites and P. falciparum gametocytes. These ILs were found to display similar or better in vitro activities than their covalent analogues, $N$-cinnamoylprimaquine derivatives, formerly developed by us. ${ }^{16}$ More relevantly, the ILs performed comparably or superior to the parent drug, primaquine. Ongoing studies in our group, involving other antimalarials and carboxylic acids, as well as additional physicochemical and in vitro assays, will hopefully provide further support to the relevance of rescuing classical drugs towards development of new ILs-APIs for antimalarial chemotherapy. In summary, ILs herein reported represent new antimalarial triple-stage hits that open a new pathway to novel lowcost antimalarial IL leads, of undeniable importance for malaria chemoprophylaxis, radical cure and containment.

\section{Acknowledgements}

Thanks are due to "Fundação para a Ciência e Tecnologia" (FCT, Portugal) for supporting Research Units GHTM and UCIBIOREQUIMTE through projects UID/Multi/04413/2013 and UID/ MULTI/04378/2013, respectively. Thanks are also due to "Comissão de Coordenação e Desenvolvimento Regional do Norte (CCDR-N)/NORTE2020/Portugal 2020" for funding through project DESignBIOtechHealth (Norte-01-0145-FEDER000024). The gametocytes screening was supported by the 
Global Health Program of the Bill \& Melinda Gates Foundation (Grant OPP1040394).

\section{Notes and references}

1 R. Ferraz, L. C. Branco, I. M. Marrucho, J. M. M. Araújo, L. P. N. Rebelo, M. N. da Ponte, C. Prudêncio, J. P. Noronha and Z. Petrovski, MedChemComm, 2012, 3, 494-497.

2 R. Ferraz, L. C. Branco, C. Prudêncio, J. P. Noronha and Z. Petrovski, ChemMedChem, 2011, 6, 975-985.

3 I. M. Marrucho, L. C. Branco and L. P. N. Rebelo, Annu. Rev. Chem. Biomol. Eng., 2014, 5, 527-546.

4 R. Ferraz, J. Costa-Rodrigues, M. H. Fernandes, M. M. Santos, I. M. Marrucho, L. P. N. Rebelo, C. Prudêncio, J. P. Noronha, Z. Petrovski and L. C. Branco, ChemMedChem, 2015, 10, 1480-1483.

5 A. Costa, A. Forte, K. Zalewska, G. Tiago, Z. Petrovski and L. C. Branco, Green Chem. Lett. Rev., 2014, 8, 8-12.

6 R. Ferraz, V. Teixeira, D. Rodrigues, R. Fernandes, C. Prudêncio, J. P. Noronha, Z. Petrovski and L. C. Branco, RSC Adv., 2014, 4, 4301-4307.

7 C. Florindo, J. M. M. Araújo, F. Alves, C. Matos, R. Ferraz, C. Prudêncio, J. P. Noronha, Z. Petrovski, L. Branco, L. P. N. Rebelo and I. M. Marrucho, Int. J. Pharm., 2013, 456, 553-559.

8 C. P. Frizzo, I. M. Gindri, A. Z. Tier, L. Buriol, D. N. Moreira and M. A. P. Martins, Pharmaceutical Salts: Solids to Liquids by Using Ionic Liquid Design, 2013.

9 World Health Organization, Malaria Report, 2015, p. 8, http:// apps.who.int/iris/bitstream/10665/200018/1/9789241565158_ eng.pdf?ua=1, accessed April 2016.

10 C. Teixeira, N. Vale, B. Pérez, A. Gomes, J. R. B. Gomes and P. Gomes, Chem. Rev., 2014, 114, 11164-11220.

11 R. Fernandes, P. Amador, C. Oliveira and C. Prudêncio, Sci. World J., 2014, 782897.

12 M. R. Cole, J. A. Hobden and I. M. Warner, Molecules, 2015, 20, 6466-6487.

13 A. Gomes, B. Pérez, I. Albuquerque, M. Machado, M. Prudêncio, F. Nogueira, C. Teixeira and P. Gomes, ChemMedChem, 2014, 9, 305-310.
14 S. Sinha, B. Medhi and R. Sehgal, Parasite, 2014, 21, 61.

15 C. Florindo, A. Costa, C. Matos, S. L. Nunes, A. N. Matias,

C. M. M. Duarte, L. P. N. Rebelo, L. C. Branco and I. M. Marrucho, Int. J. Pharm., 2014, 469, 179-189.

16 B. Pérez, C. Teixeira, I. S. Albuquerque, J. Gut, P. J. Rosenthal, M. Prudêncio and P. Gomes, MedChemComm, 2012, 3, 1170-1172.

17 B. C. Pérez, C. Teixeira, I. S. Albuquerque, J. Gut, P. J. Rosenthal, J. R. B. Gomes, M. Prudêncio and P. Gomes, J. Med. Chem., 2013, 56, 556-567.

18 J. Wiesner, A. Mitsch, P. Wissner, H. Jomaa and M. Schlitzer, Bioorg. Med. Chem. Lett., 2001, 11, 423-424.

19 B. Meunier, Acc. Chem. Res., 2008, 41, 69-77.

20 K. Bica, C. Rijksen, M. Nieuwenhuyzen and R. D. Rogers, Phys. Chem. Chem. Phys., 2010, 12, 2011-2017.

21 M. R. Cole, M. Li, B. El-Zahab, M. E. Janes, D. Hayes and I. M. Warner, Chem. Biol. Drug Des., 2011, 78, 33-41.

22 K. Rishikesh, A. Kamath, M. H. Hande, S. Vidyasagar, R. V. Acharya, V. Acharya, J. Belle, A. B. Shastry and K. Saravu, Malar. J., 2015, 14, 310.

23 M. L. Sykes and V. M. Avery, J. Med. Chem., 2013, 56, 77277740.

24 A. P. Gorka, L. M. Jacobs and P. D. Roepe, Malar. J., 2013, 12, 332.

25 M. Prudêncio, M. M. Mota and A. M. Mendes, Trends Parasitol., 2011, 27, 565-574.

26 I. H. J. Ploemen, M. Prudêncio, B. G. Douradinha, J. Ramesar, J. Fonager, G.-J. van Gemert, A. J. F. Luty, C. C. Hermsen, R. W. Sauerwein, F. G. Baptista, M. M. Mota, A. P. Waters, I. Que, C. W. G. M. Lowik, S. M. Khan, C. J. Janse and B. M. D. Franke-Fayard, PLoS One, 2009, 4, e7881.

27 S. D'Alessandro, G. Camarda, Y. Corbett, G. Siciliano, S. Parapini, L. Cevenini, E. Michelini, A. Roda, D. Leroy and D. Taramelli, J. Antimicrob. Chemother., 2016, dkv493.

28 Molecular Operating Environment (MOE), 2015.1001; Chemical Computing Group Inc., 1010 Sherbooke St. West, Suite \#910, Montreal, QC, Canada, H3A 2R7, 201. 